\title{
El Six Sigma como técnica de modelo de gestión
}

\author{
Six Sigma as a management model technique
}

1 Ubaldo Olmedo Saldarriaga Coronel Universidad Cesar Vallejo, Piura, Perú. P7002323028@ucvvirtual.edu.pe

2 Cecilia Teresita de Jesús Carbajal Llauce Universidad Cesar Vallejo, Piura, Perú. cllaucect@ucvvirtual.edu.pe
(iD) https://orcid.org/0000-0003-4247-0484

D https://orcid.org/0000-0002-1162-8755

Artículo de Investigación Científica y Tecnológica

Enviado: 16/12/2021

Revisado: 27/12/2021

Aceptado: 28/01/2021

Publicado:05/02/2022

DOI: https://doi.org/10.33262/ap.v4i1.1.148

Saldarriaga Coronel, U. O., \& Carbajal Llauce, C. T. de J. (2022). El Six Sigma como técnica de modelo de gestión . AlfaPublicaciones, 4(1.1), 94-108.

Cítese: https://doi.org/10.33262/ap.v4i1.1.148

ALFA PUBLICACIONES, es una Revista Multidisciplinar, Trimestral, que se publicará en soporte electrónico tiene como misión contribuir a la formación de profesionales competentes con visión humanística y crítica que sean capaces de exponer sus resultados investigativos y científicos en la misma medida que se promueva mediante su intervención cambios positivos en la sociedad. https://alfapublicaciones.com

La revista es editada por la Editorial Ciencia Digital (Editorial de prestigio registrada en la Cámara Ecuatoriana de Libro con No de Afiliación 663) www.celibro.org.ec 


\section{Palabras}

claves:

gestión, calidad, Six Sigma, DMAIC, técnica, procesos

\section{Keywords:}

management, quality, Six

Sigma, DMAIC, technique, processes

\section{Resumen}

Hoy en día, las empresas buscan optimizar sus procesos, con el propósito de disminuir perdidas, tanto en la materia prima como a nivel financiero, así como también, para la mejora y optimización de los servicios, para ello buscan aplicar técnicas y herramientas de gestión de calidad que le permitan conocer, en cuales de los procesos que se llevan a cabo están presentando las principales fallas o errores. En el mercado existe diversidad de estas técnicas, por lo que las organizaciones pueden escoger aquellas que más se adecuan o se adaptan a sus necesidades particulares. Una de estas técnicas es el Six Sigma, es una herramienta que actualmente está siendo aplicada por muchas empresas, en vista de los buenos resultados que ha generado en aquellas que ya lo han hecho. Esta técnica permite determinar las causas que originan los errores, así como defectos y/o los retrasos en cada uno de los procesos que se requieran evaluar, a través de la aplicación de cinco fases: Definir, Medir, Analizar, Mejorar y Controlar. Finalmente, como conclusión se menciona que esta técnica permitirá medir si los productos o servicios que se ofrecen son de alta calidad y satisfacen las necesidades del cliente, su uso se hace necesario si las organizaciones buscan evaluar y mejorar sus procesos, ya que permite la reducción de la complejidad en los diseños de productos y procesos.

\section{Abstract}

Today, companies seek to optimize their processes, in order to reduce losses, both in raw materials and financially, as well as, for the improvement and optimization of services, for this they seek to apply management techniques and tools of quality that allow you to know, in which of the processes that are carried out are presenting the main failures or errors. In the market there is a diversity of these techniques, so that organizations can choose those that best suit or adapt to their particular needs. One of these techniques is Six Sigma, it is a tool that is currently being applied by many companies, in view of the good results it has generated in those that have already done so. This technique allows to determine the causes that originate the errors, as well as defects and / or delays in each of the processes that need to be evaluated, through the application of five phases: Define, Measure, Analyze, Improve and Control. Finally, as a conclusion, it is mentioned that this technique will allow to measure if the products or services offered are of high 
quality and satisfy the needs of the client, its use is necessary if organizations seek to evaluate and improve their processes, since it allows the reduction of complexity in product and process designs.

\section{Introducción}

El mundo empresarial, se encuentra actualmente afrontando grandes cambios en todos los ámbitos, tecnológicos, económicos, financieros, entre otros aspectos, por ello, buscan alternativas para adecuarse a estos cambios y que le permitan continuar con su operatividad, y por ende cumplir tanto con sus metas organizacionales como las de tipo financiero.

Por esta razón, las organizaciones se centran en buscar la excelencia en cuanto a la gestión de calidad, con miras a mejorar y perfeccionar sus procesos, que le permitan generar productos y/o servicios con calidad y que satisfagan los requerimientos y expectativas del cliente.

Según lo mencionado en la investigación de Guevara-Mosquera (2020), los principales aportes a la gestión de calidad fueron realizados por Edward Deming y Joseph Juran en 1950, quienes implementaron el control estadístico de procesos, con el propósito de lograr una alta efectividad en las operaciones. Asimismo, define la gestión de calidad, como un proceso que se lleva a cabo de forma dinámica e interactiva que se fundamenta en planear, organizar, liderar y controlar, todas las actividades de una organización, a fin de cumplir con los objetivos fijados y satisfacer tanto a los clientes como a todos los que se involucran con ella.

Por otra parte, Ordinola (2017), menciona que la gestión de calidad son aquellos procesos a través de los cuales las organizaciones pueden alcanzar la obtención de un excelente producto o servicio, procesos que resultan de gran importancia, dado a que son estos los que permitirán que sus productos o servicios sean aceptados y aprobados en aquellos mercados donde se comercialicen.

Dado al concepto de la gestión de calidad, se puede indicar que, a través de la implementación de estos procesos, las organizaciones buscan optimizarlos, no tanto para alcanzar las metas económicas, sino también, para lograr la captación de nuevos clientes y mantener los que ya consumen sus productos o servicios, de manera tal que se pueda lograr su estabilidad, crecimiento y expansión.

Es por ello, que cada día se investigan sobre los distintos modelos de gestión existentes, lo cual les permite a las organizaciones escoger aquel que más se adapte a sus necesidades y de esta forma conseguir los resultados fijados y deseados. 
Una de estas técnicas de gestión es el Six Sigma, la cual están usando muchas empresas y que han logrado alcanzar la meta fijada, dado a que esa permite buscar la excelencia en la producción de bienes y servicios.

En este sentido, Facho (2017) señala, que la estrategia del Six Sigma inició en 1987, por Bob Galvin quien se desempeñada como directivo de la empresa Motorola, quien implanta la técnica, con el propósito de disminuir la cantidad de defectos en los productos electrónicos, logrando obtener resultados mayores a los esperados, lo cual origino su uso en el mundo empresarial y académico, siendo adoptada por otras grandes compañías como: Allied Signal en 1994 y General Electric en 1995, obteniendo importantes resultados en sus operaciones, y logrando economizar gastos.

Según lo mencionado por Orellana (2021), las empresas Motorola y General Electric, tuvieron en común un alto compromiso y liderazgo de todos los directivos, por lo cual lograron estos resultados:

- Motorola logro un ahorro de aproximadamente 1000 millones de dólares en un lapso de tres años alcanzado el premio a la calidad Malcom Baldrige.

- General Electric, logo un ahorro de 2570 millones dólares en un lapso de tres años.

Según Pons et al. (2018), el Six Sigma es una técnica cuyo propósito es minimizar la variación de los procesos a fin de optimizar la calidad. Sigma en estadísticas, significa la desviación típica de un grupo de datos, es decir, la disgregación de los valores en relación a la media. Este valor sigma, se relaciona de forma directa con el número de unidades defectuosas, de forma tal que si la desviación presentada es pequeña se obtendrán pocas unidades defectuosas. Ajustando esta conceptualización a la definición a la metodología Six Sigma, se determina que el objetivo es implantar un proceso en el cual minimice los defectos y se pueda obtener como máximo 3,4 piezas defectuosas por millón.

Por otra parte, Navarro et al. (2017), señalan que el Six Sigma, constituye el número de desviaciones estándar que se obtienen al final de un proceso. Su objetivo primordial, es aumentar la capacidad de los procesos, con el propósito de que se generen un mínimo de defectos por cada millón de unidades producidas.

En este mismo orden de ideas Panta \& Espinoza (2017), definen el Six Sigma como una medida de la calidad cuyo propósito es buscar la perfección, la cual se lleva a cabo a través de datos, que busca la eliminación de defectos, además de mostrar cuantitativamente como se está realizando un proceso.

Entonces, queda claro, que, con la implementación de esta técnica, las empresas buscan minimizar los defectos o errores durante la producción de cualquier producto, servicio o 
simplemente mejorar un proceso administrativo, con el propósito de optimizar sus procesos y obtener los resultados deseados.

De esta manera el objetivo de esta investigación se basa en analizar el funcionamiento de la técnica de gestión Six Sigma como elemento importante dentro de las organizaciones. La metodología aplicada es de diseño bibliográfico de tipo documental.

\section{Metodología}

Para la realización de esta investigación, se basó en la metodología de la investigación documental, a través de la revisión bibliográfica, de documentos de carácter científico, artículos, publicaciones académicas, tendencias en el mercado, medios electrónicos, de diversas índoles, tomando las ideas y concepto adoptadas por los autores, y realizar un análisis descriptivo para el desarrollo del tema y desarrollar de manera clara y sencilla los aspectos más relevantes sobre la técnica de gestión Six Sigma.

La Técnica del Six Sigma, es usada desde los años 80, y es una técnica que busca la excelencia en los procesos de las organizaciones, a fin de disminuir al mínimo el margen de error o defecto en los productos y/o servicios que están generan. Actualmente el uso de esta técnica se ha incrementado a nivel mundial, dado a los buenos resultados que esta genera para las organizaciones.

\section{Resultados y Discusión}

\section{Principios del Six Sigma}

Según lo mencionado por Lefcovich (2015, citado por Zuluaga, 2016), los principios que conducen la metodología Six Sigma son los mostrados en la tabla 1

\section{Tabla 1}

Principios de la Metodología Six Sigma

\begin{tabular}{cl}
\hline N. $^{\mathbf{2}}$ & \multicolumn{1}{c}{ Principio } \\
\hline 1 & $\begin{array}{l}\text { Su enfoque va dirigido al cliente, siendo la prioridad principal la satisfacción } \\
\text { de este. }\end{array}$ \\
2 Orientada a datos y hechos, los cuales son analizados e interpretados \\
\\
Los procesos se ubican donde se encuentra la acción. Reunir todas las energías \\
y métodos de análisis en los procesos a fin de mejorarlos, con el propósito de \\
satisfacer a los clientes, tantos internos como externos, haciendo un uso \\
eficiente de los recursos y dando una respuesta rápida e inmediata.
\end{tabular}




\section{Tabla 1}

Principios de la Metodología Six Sigma (continuación)

\begin{tabular}{|c|c|}
\hline N. ${ }^{\circ}$ & Principio \\
\hline 4 & $\begin{array}{l}\text { Dirección proactiva: Ser proactivo es intervenir con anticipación ante cualquier } \\
\text { situación. De allí a que es necesario prever que puede salir mal, para tomar } \\
\text { medidas y acciones que eviten los errores y fallas. }\end{array}$ \\
\hline 5 & $\begin{array}{l}\text { Colaboración sin barreras. Toda la organización debe estar comprometida con } \\
\text { la implementación de la herramienta, con el propósito de alcanzar las mejoras } \\
\text { y el incremento de los niveles de calidad. Se apoya en un equipo competente y } \\
\text { calificado, donde cada uno tiene asignado el rol y la responsabilidad para llevar } \\
\text { a cabo el proyecto. }\end{array}$ \\
\hline 6 & $\begin{array}{l}\text { Buscar la perfección y tolerar los fallos. A través de esta metodología se busca } \\
\text { la generación de ideas nuevas e innovadora, sin embargo, estas pueden } \\
\text { presentar fallas o no llegan a cumplir las metas deseadas, por lo cual se debe } \\
\text { aceptar que no siempre las ideas resultan tal y como se planean, por lo cual es } \\
\text { importante que los resultados negativos también sean aceptados y corregidos, a } \\
\text { fin de no bloquear la generación de nuevas ideas que estén destinadas a la } \\
\text { mejora de los procesos. }\end{array}$ \\
\hline 7 & $\begin{array}{l}\text { Cambio cultural orientado a implementar el empowerment. Se sustenta en que } \\
\text { los directivos de la organización apoyen a los trabajadores, a fin de lograr un } \\
\text { mayor grado de satisfacción al cliente, generando confianza en todos los } \\
\text { niveles, con el propósito de que los trabajadores puedan notificar fallas o } \\
\text { problemas presentados que afecta el servicio al cliente. }\end{array}$ \\
\hline 8 & $\begin{array}{l}\text { Estimular la generación de ideas, para ponerlas en marcha y contribuir a mejorar } \\
\text { el rendimiento y performance de la organización. La puesta en marcha de los } \\
\text { proyectos genera ahorro y aumentos en la utilidad. }\end{array}$ \\
\hline 9 & $\begin{array}{l}\text { La existencia de un liderazgo comprometido, a fin de que los cambios y toma } \\
\text { de decisiones sean asumidos por toda la empresa en todos los niveles, } \\
\text { comenzando por el líder de la organización. }\end{array}$ \\
\hline
\end{tabular}

Fuente: Orellana (2021), Zuluaga (2016)

Queda claro, que los principios fundamentales de la metodología Six Sigma, es la orientación a satisfacer las necesidades y expectativas del cliente, como principal razón en la producción o generación de servicios, así como también, generar un ambiente organizacional que involucre a todos los integrantes de la organización a entender, manejar y poner en marcha la herramienta, y de esta forma lograr con las metas fijadas. 


\section{Características}

Calderón \& Orozco (2020), señalan que el Six Sigma presenta tres características que las diferencias de otras técnicas o herramientas que también están enfocadas a la calidad, y se muestran en la tabla 2.

\section{Tabla 2}

\section{Características del Six Sigma}

\begin{tabular}{cl}
\hline N. $^{{ }^{\circ}}$ & \multicolumn{1}{c}{ Características } \\
\hline 1 & El Six Sigma está en un 100\% enfocado en el cliente y en satisfacer sus \\
& necesidades, lo cual estimula la mejora continua. \\
2 & La puesta en marcha de los proyectos hace retornar las inversiones \\
& Diseña métodos para que la organización se maneje de forma diferente pero \\
3 & orientada a la eficacia, involucrando desde la alta dirección hasta los \\
& trabajadores de más bajo nivel, por lo cual todos deben de tener conocimientos \\
& sobre la herramienta y poder alcanzar magnos resultados
\end{tabular}

Fuente: Calderón \& Orosco (2020)

\section{Ventajas del Six Sigma}

Falero \& Tafur (2021), menciona que, implementar Six Sigma significa una situación de ganar-ganar, puesto que a través de esta se crean estrategias y un adecuado ambiente para el flujo y la eliminación de desperdicios. Menciona como ventajas de esta metodología.

- Eliminación del desperdicio: En todas las organizaciones se generan desperdicios, que no es otra cosa, que aquellos productos o servicios que no cumplen con los parámetros deseados, lo cual genera pérdidas de tiempo, dañando a todo el sistema.

- Una Mejor Calidad: a través de Six Sigma, se logra una mejor calidad, y por ende se obtienen mejores resultados, minimizando los defectos en cada proceso, lo cual se traduce en un producto y servicio de buena calidad.

- Identifica la causa raíz: por medio de la técnica análisis de causa raíz, que está incluida en la metodología Sig Sixma, se puede analizar con precisión el problema que se está generando y conocer lo que lo causa, lo que permite implementar los lineamientos para obtener la respectiva solución.

- Satisfacción del cliente: el Six Sigma cuenta con VOC (Voice of the Customer), que es una herramienta que se centra en localizar las necesidades y deseos de sus clientes, cuyo propósito es poder llenar las expectativas y requerimientos y permita un alto grado de satisfacción a los clientes. 
- La mejora del proceso: a través de la metodología Six Sigma, se pueden mejorar todos los procesos en cualquier organización, aplicando el método DMAIC, a través del cual muchas de ellas han alcanzado su éxito.

\section{Roles del equipo Six Sigma}

Para Rincón (2019), los principales roles y funciones en la metodología Six Sigma son:

- Champion (Campeón): Son aquellos individuos de la organización, que tienen como objetivo guiar las iniciativas de Six Sigma. Por ello deben identificar y apoyar los proyectos cuyos resultados se estiman puedan generar algún impacto, así como colaborar en la visión estrategia de la organización. De igual manera, deben proporcionar dirección y visión del proyecto, así como también tienen la capacidad de conectar los proyectos con las estrategias de la organización.

- Master Black Belt (Maestro de Cinta Negra): Este es la persona experta en la metodología de Six Sigma, el cual cuenta con conocimientos tantos teóricos como prácticos muy avanzados, está capacitado para suministrar el adiestramiento de la metodología. Entre sus responsabilidades están: coordinar e implementar la metodología en la organización, desde la alta directiva (Black Belts y Green Belts) hasta los empleados. En algunos casos pueden llegar a liderar proyectos de alta envergadura, sin embargo, suelen dedicarse al desarrollo de los Black Belts.

- Black Belt (Cinta Negra): Son los que lideran los proyectos generalmente a tiempo completo. Poseen y manejan los conceptos de la metodología cabalmente. Trabajan en conjunto con los champions, los miembros del proyecto y cualquier persona que forma parte de estos. Su papel es de gran importancia ya que cumple la función de la divulgación del conocimiento de la metodología, así como actuar como tutor de los Green Belts. Generalmente el entrenamiento tarda alrededor de 20 días y para el desarrollo puede ir desde 18 a 24 meses.

- Green Belt (Cinta Verde): Este personal cuenta con buenos conocimientos tanto de las herramientas como de la metodología de Six Sigma, sin embargo, en menor proporción que los Black Belt, en algunas oportunidades pueden llegar a liderar proyectos o simplemente ser miembros del equipo de trabajo de uno de ellos. Su capacitación puede tardar de 8 a 10 días.

\section{Etapas del Six Sigma}

Como se ha mencionado previamente, la metodología Six Sigma, busca la reducción de los defectos y errores en todos los procesos de una organización, minimizando los costos lo cual se traduce en la obtención de mayores ganancias. 
Para llevar a cabo la metodología es necesario realizar de forma ordenada una serie de fases, las cuales según lo mencionado por Ramos (2017), estas fases son: Definir el proceso, medirlo, analizar sus datos, mejorarlo y controlarlo, formando las iniciales de estas etapas el nombre abreviado de DMAIC como se observa en la figura 1.

\section{Figura 1}

Etapas del Six Sigma

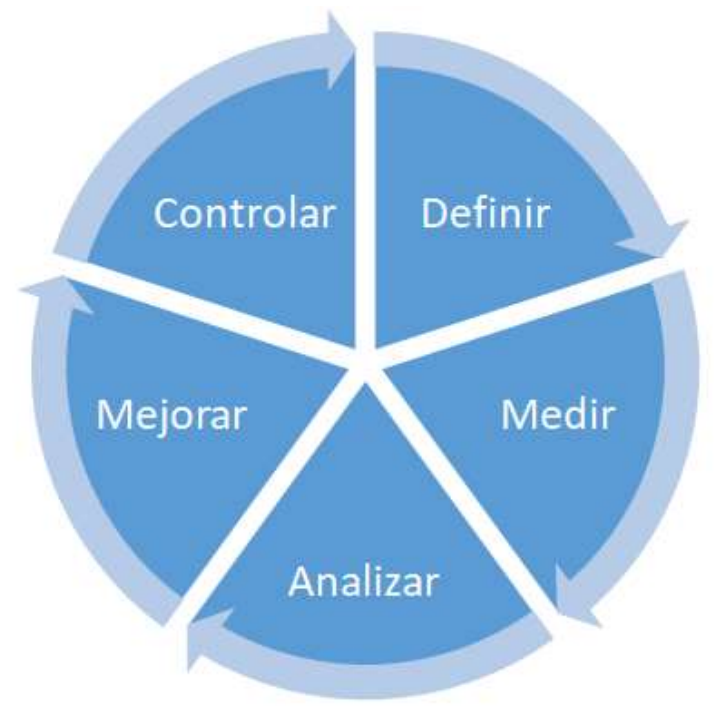

En este mismo orden de ideas Ramos (2017), señala cada una de las etapas tal como se describe a continuación:

- Definir: En esta primera fase se deben identificar cuales proyectos deben ser evaluados por la organización a través de la metodología Six Sigma, es una etapa de gran importancia ya que pueden evitarse el uso inadecuado de recursos. Posteriormente se procede a preparar y seleccionar el equipo de trabajo para llevarlo a cabo.

Las herramientas más usadas para la realización de esta fase son:

a) Diagrama de flujo de procesos

b) Diagrama de causa-efecto o de espina de pescado o de Ishikawa

c) Diagrama de Pareto

d) Histogramas

e) Gráficos de tendencia

Calderón \& Orozco (2020), señala que esta fase se divide en seis etapas:

a) Identificar tanto los clientes, internos como externos

b) Establecer las diatribas de la calidad del proyecto 
c) Seleccionar el problema, darle prioridad a aquel que está causando mayor daño

d) De qué forma el problema está impactando a la organización

e) Describir detalladamente el problema

f) Definir y especificar el alcance que tendrá el proyecto

- Medir: Esta fase consiste determinación del proceso, para lo cual se deben identificar cuáles serán los requerimientos claves de los clientes, todas las características claves del producto (variables del resultado) y aquellos parámetros (variables de entrada), que pueden afectar la marcha del proceso y sus características o variables clave.

Las herramientas más usadas para llevar a cabo esta fase son:

a) Modelación de las características de calidad

b) Evaluación de la normalidad de los datos

c) Evaluación de la exactitud y linealidad

d) Evaluación de la repetitividad y la reproducibilidad

e) Modos alternativos de la evaluación de los sistemas de medición

f) Análisis de fiabilidad

De igual manera Calderón \& Orozco (2020), indican que el objetivo de esta fase es medir el tamaño del problema, a fin de aplicar mejoras y obtener una solución. Divide esta fase en las siguientes etapas que son:

a) Realizar una selección de las críticas a la calidad del proceso

b) Constituir y validar un plan que permita la recolección de datos

c) El uso de estadística básica, sea descriptiva o inferencial, también pueden usarse otras medidas tales como la tendencia central y la de dispersión.

- Analizar: En esta fase, el equipo debe evaluar todos los datos tanto de los resultados actuales como de los históricos. Se amplían y verifican las hipótesis que puedan determinar las causas y efectos. Para ello es necesario el uso de herramientas estadísticas, lo cual permite que el equipo a cargo pueda corroborar los determinantes del proceso, en otras palabras, identificar aquellas variables claves de entrada que afectan las variables de prepuesta del proceso.

Las herramientas usadas en esta fase son:

a) Análisis exploratorio de datos

b) Ajuste de distribuciones

c) Contraste de hipótesis

d) Intervalos de confianza

e) Capacidad de los procesos 
- Mejorar: Es esta etapa el equipo de trabajo debe procurar establecer la causaefecto (concordancia matemática entre las variables de entrada y las variables de repuesta que sean importantes) con el propósito de poder pronosticar, mejorar y perfeccionar la puesta en marcha del proceso. Asimismo, se debe establecer el rango operacional de las variables o medidas de entrada del proceso

En esta fase se usan las siguientes herramientas:

a) Análisis de correlaciones

b) Regresión simple

c) Regresión múltiple

d) Análisis de la varianza unifactorial

e) Análisis de la varianza multifactorial

f) Modelos de series temporales

- Controlar: en esta fase se diseñan y documentan aquellos controles que se crean necesarios, a fin de certificar que lo logrado a través del Six Sigma se conserven al haber efectuado los cambios.

Las herramientas usadas en esta fase son:

a) Control estadístico de procesos

b) Control de fabricación

c) Gráficos de Shewhart

d) Curva característica de operación

e) Gráfico de control de variables

f) Gráfico de control por atributos

g) Análisis de capacidad mediante gráficos de control

h) Control estadístico de procesos / Control de aceptación

i) Muestreo por atributos

j) Muestreo por variables

\section{Herramientas usadas por la metodología Six Sigma}

En la investigación realizada por Villacrés \& Villanueva (2019), mencionan como principales herramientas de esta metodología, las siguientes:

- SIPOC: Representa a los proveedores, Entradas (Inputs), Proceso, Salida (Output) y Clientes. Es un diagrama el cual permite obtener una visualización para comprender un determinado proceso. Este diagrama es realizado con la colaboración del equipo de trabajo a través de la generación de ideas y discusiones. 
- Voz del Cliente (VOC): Dentro de esta herramienta se encuentra el uso de entrevistas, grupos focales, buzones de sugerencias, entre otras, las cuales permiten obtener información sobre los requerimientos y puntos de vista de los clientes. A través del análisis de Kano, se pueden cambiar los datos brutos obtenidos en datos cualitativos y cuantitativos.

- Matriz Causa Efecto: Se usa principalmente para calcular los efectos a fin de computar las puntuaciones que son utilizadas para clasificar las causas y la medición de la matriz que se usa para escoger las entradas.

- Plan de Recolección de Datos: Consiste en la recolección de datos, los cuales deben proveer la información necesaria para la realización del proyecto, los cuales deben permitir determinar, el tamaño de la muestra, identificar las fuentes de datos, utilización de hojas de recolección de datos y la asignación al equipo de las mismas.

- Grafica de Pareto: Está representada por una gráfica de barras, y su eje horizontal detalla las categorías, se enfoca en los problemas que son considerados más importantes por resolver.

- Graficas de dispersión: Está representada por una gráfica donde dos variables se trazan, permitiendo una visualización de como las variables se corresponden entre sí.

- Diagrama de Ishikawa: Esta herramienta permite a través de un dibujo que simula un esqueleto de un pescado, plasmar las causas del problema, así como los efectos que estos producen.

- Análisis de Regresión: Esta herramienta se considera como el equivalente matemático del diagrama de dispersión. Se representa a través de una ecuación, la cual permite formular la dependencia de una variable con uno o más de estas.

- Control Estadísticos de Procesos: con esta herramienta se preparan las gráficas de control, las cuales muestran las capacidades que se han mejorado en el proceso.

El estudio de la teoría, sobre la metodología Six Sigma, permite entender, que es una técnica que permite a cualquier organización implementarla a fin de mejorar sus procesos internos, bien en los procesos relacionados directamente con la producción (de bienes o servicios), o para mejorar y optimizar los procedimientos administrativos.

Es importante que, al momento de implementar el uso de esta técnica, la organización este comprometida en la aplicación y ejecución de la misma, entendiéndose que todo cambio genera inversión de capital, por lo cual, se debe asegurar con la disponibilidad financiera para el inicio del uso de la técnica. 
De igual manera, se debe contar con un equipo capacitado en el manejo de la herramienta, el cual será el responsable de dar apoyo a todos los que así lo requieran dentro de la organización.

Otros de los aspectos que también deben ser considerados es contar con el apoyo total tanto de la alta gerencia como de todos los miembros de la organización, y entender que el uso de la técnica permitirá perfeccionar y mejorar los procesos, siendo necesario formar y capacitar a todos los empleados que intervendrán en la puesta en marcha, a fin de que cuenten con los conocimientos y características necesarias para implementar la técnica.

\section{Conclusiones}

- El Six Sigma permitirá medir si los productos o servicios que se ofrecen son de alta calidad y satisfacen las necesidades del cliente. Su uso se hace necesario si las organizaciones buscan evaluar y mejorar sus procesos, ya que permite la reducción de la complejidad en los diseños de productos y procesos.

- Asimismo, es importante que las organizaciones se enfoquen en mejorar aquellos procesos que están originando realmente un problema y que necesita mejorar.

- Por otro lado, la recolección de datos e información, así como la veracidad de los mismos, permitirán obtener un mejor resultado, ya que, a través de estos, se podrá conocer realmente la o las causas que generan el problema, y de esta forma proceder atacarlos para conseguir los resultados deseados.

- Dado a que esta técnica se maneja a través de la estadística y es la base para lograr el control y gestión de los procesos, se convierte en una guía para poder identificar aquellas variables realmente críticas y que afectan la calidad y los procesos.

- Otro de los aspectos relevantes de esta técnica, es que a través de ella pueden ser evaluados los resultados obtenidos y por ende cuantificar el impacto.

\section{Referencias Bibliográficas}

Calderón, Y., \& Orosco, R. (2020). Implementación de la herramienta de Six Sigma para mejorar la productividad en la Empresa Manufacturas Textiles Vialniva S.A.C, Carabayllo 2020. Lima, Perú: Trabajo especial de grado de la Universidad Cesar Vallejo para optar por el título de Ingeniero Industrial.

Facho, G. (2017). Mejora de procesos en una empresa textil exportadora mediante la metodología Six Sigma. Lima, Perú: Trabajo especial de grado de la Universidad Nacional Mayor de San Marcos para optar al título de Ingeniería Textil y Confecciones. 
Falero, E., \& Tafur, G. (2021). Implementación de la metodología Six Sigma para mejorar la productividad de la empresa American Mecanic Motors E.I.R.L, 2020. Trabajo especial de grado de la Universidad Privada del Norte.

Guevara-Mosquera, N. (2020). Metodología Six Sigma para la mejora de la calidad en la Empresa Reproimav, Ecuador. Ecuador: EmTHYMOS Revista de estudios empresariales 1(1).

Navarro, E., Soler, V., \& Pérez, A. (2017). Metodología e Implementación de Six Sigma. 3C Empresa Investigación y Pensamiento Crítico, 73-80.

Ordinola, D. (2017). Caracterización de la gestión de calidad con el uso del marketing en el restaurante "Perla del Chira", del distrito de Marcavelica, provincia de Sullna, año 2017. Perú: Trabajo especial de grado de la Universidad Católica los Ángeles de Chimbote.

Orellana, E. (2021). Optimización del proceso de soporte técnico de fibra óptica basado en la metodología Seis Sigma. Caso: empresa de servicios de telecomunicaciones. Quito, Ecuador: Trabajo especial de grado de la Pontificia Universidad Católica del Ecuador para optar al título de Master en Administración de Empresas con mención en Calidad y Productividad.

Panta, O., \& Espinoza, J. (2017). Propuesta de mejora del proceso de cobranza de energía eléctrica en una cadena de centros comerciales. Lima: Trabajo especial de grado de la Universidad Peruana de Ciencias Aplicadas para optar al título de Ingeniero Industrial.

Pons, B., Soler, V., \& Pérez, A. (2018). Metodología Six Sigma. Comparación entre ciclo PDCA y DMAIC. Cuadernos de Investigación Aplicada, 27-34.

Ramos, M. (2017). Propuesta de aplicación de AMEF potencializado con Six Sigma en la red de distribución de CFE en la zona Tehuantepec. Xalapa, Veracruz: Trabajo especial de grado de la Universidad Veracruzana Campus Xalapa para optar al grado de Maestro en Ingeniería de la Calidad.

Rincón, B. (2019). Implementación del método six sigma para la mejora de la operación Logística y el cumplimiento de los indicadores operativos de la empresa OPAV sede Girón. Bucaramanga, Colombia: Trabajo especial de grado de la Universidad Pontificia Bolivariana para optar al título de Ingeniero Industrial.

Villacrés, L., \& Villanueva, D. (2019). Aplicación de la metodología Six Sigma para mejorar el proceso de registro y control de asistencia en el proyecto especial CORAH 2019. Pucallpa, Perú: Trabajo especial de grado de la Universidad Privada de Pucallpa para optar al título de Ingeniero de Sistemas. 
Zuluaga, P. (2016). Aplicación de la metodología Six Sigma para solucionar problemas de calidad en una empresa metalmecánica. Medellín, Colombia: Trabajo especial de grado de la Universidad de Medellín.

Ciencia Digital

El artículo que se publica es de exclusiva responsabilidad de los autores y no necesariamente reflejan el pensamiento de la Revista Alfa Publicaciones.

\section{Liencia}

El artículo queda en propiedad de la revista y, por tanto, su publicación parcial y/o total en otro medio tiene que ser autorizado por el director de la Revista Alfa Publicaciones.
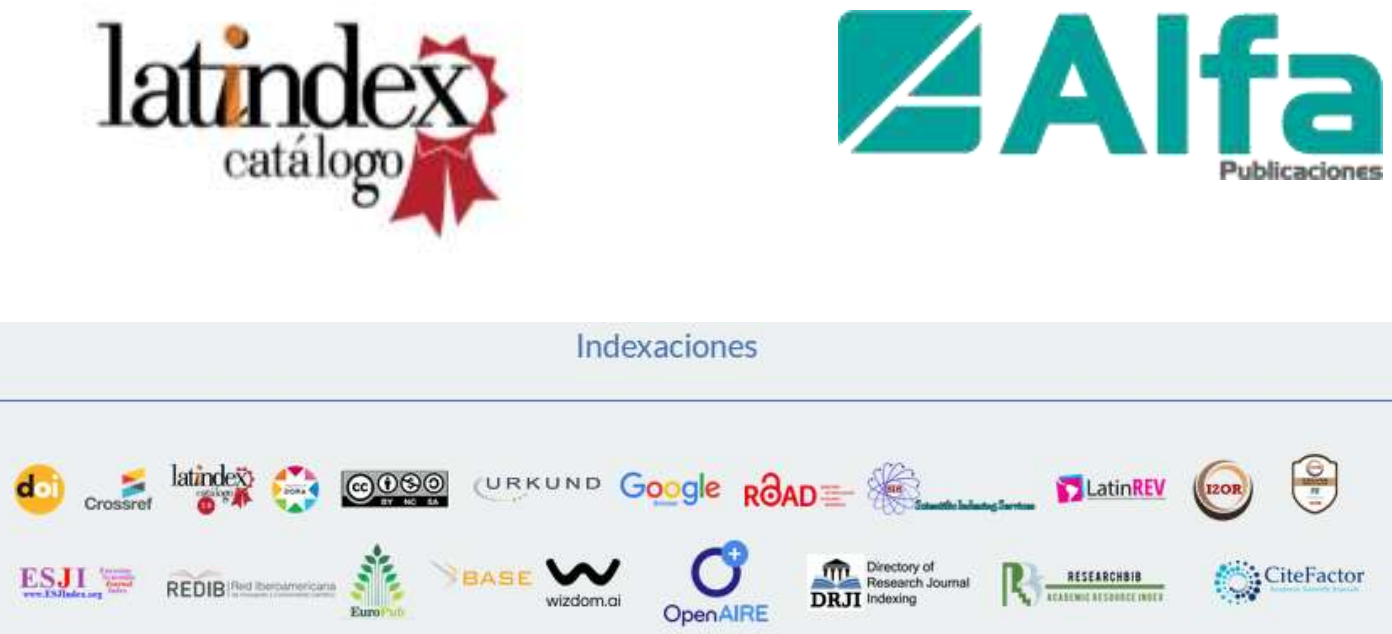